06

\title{
Эволюция интенсивных световых импульсов в нелинейной среде с учетом эффекта Рамана
}

\author{
(C) C.К. Иванов ${ }^{1,2}$, A.M. Камчатнов ${ }^{1,2}$ \\ ${ }^{1}$ Институт спектроскопии РАН, \\ 108840 Троицк, Москва, Россия \\ ${ }^{2}$ Московский фризико-технический институт, \\ 141700 Долгопрудный, Московская обл., Россия \\ e-mail: ivanoff.iks@gmail.com
}

Поступила в редакцию 25.02.2019 г.

В окончательной редакции 25.02.2019 г.

Принята к публикации 15.03.2019 г.

Исследована эволюция интенсивных световых импульсов в нелинейных одномодовых световодах, динамика света в которых описывается нелинейным уравнением Шрёдингера с рамановским членом, обусловленным вынужденным комбинационным саморассеянием света. Показано, что при эволюции достаточно интенсивных импульсов образуются дисперсионные ударные волны, поведение которых гораздо более многообразно, чем в случае обычного нелинейного уравнения Шрёдингера с керровской нелинейностью. В предположении малости рамановского члена, рассматриваемого как возмущение, получены уравнения Уизема, описывающие медленную эволюцию дисперсионных ударных волн. Показано, что при учете рамановского эффекта дисперсионные ударные волны могут асимптотически приобретать стационарный профиль. Аналитическая теория подтверждена численными расчетами.

Ключевые слова: нелинейная среда, эффект Рамана, солитоны, дисперсионные ударные волны, уравнения модуляции Уизема.

DOI: $10.21883 /$ OS.2019.07.47936.87-19

\section{1. Введение}

Проблема эволюции световых импульсов в волноводах является областью активных современных экспериментальных и теоретических исследований. Известно, что решение этой задачи в приближении, описывающем динамику огибающей электрического поля светового импульса без учета дисперсии среды, приводит к „опрокидыванию“ волны на конечной длине световода. В точке опрокидывания первые производные огибающей импульса по координате или времени становятся бесконечными, а формальное решение уравнений бездисперсионного приближения после точки опрокидывания оказывается многозначным и теряет свой физический смысл. Учет дисперсии устраняет такое нефизическое поведение, но эволюционные уравнения для огибающих приобретают при этом производные более высокого порядка, что весьма затрудняет их аналитическую трактовку. Тем не менее из численных экспериментов выясняется следующая качественная картина явления. После опрокидывания волны вместо области многозначности образуется расширяющаяся область быстрых нелинейных осцилляций, параметры огибающей в которой изменяются медленно по сравнению с характерной частотой колебаний и их длиной волны. Эта область быстрых осцилляций получила название ,дисперсионной ударной волны“ (ДУВ). В нелинейной оптике такие структуры наблюдались уже давно [1,2], однако еще раньше подобные явления изучались в динамике волн на воде [3] и в физике плазмы [4]. Общая природа этого явления, возникающего вследствие совместного действия нелинейных и дисперсионных эффектов с учетом малой вязкости, была указана Р. 3. Сагдеевым [5], где вследствие вязкости ДУВ являются стационарными волновыми структурами. Общий подход к проблеме теоретического описания ДУВ в пренебрежении диссипативными эффектами был сформулирован А.В. Гуревичем и Л.П. Питаевским в работе [6] на основе теории модуляций нелинейных волн Уизема $[7,8]$, так что в этом приближении ДУВ представляется в виде эволюционирующей промодулированной нелинейной волны, являющейся периодическим решением типа бегущей волны для рассматриваемого нелинейного волнового уравнения. К настоящему времени теория и экспериментальные исследования ДУВ получили большое развитие, распространившись на другие области нелинейной физики, включая динамику нелинейных волн в бозе-эйнштейновском конденсате (см., например, обзорную статью [9] и ссылки в ней). В частности, теория ДУВ в применении к нелинейному уравнению Шрёдингера (НУШ) для сред с нормальной дисперсией и керровской нелинейностью $[10,11]$ прекрасно согласуется с экспериментальными исследованиями эволюции специальным образом сформированных импульсов прямоугольной формы в оптических световодах [12].

Хотя теория НУШ является одним из основных способов описания волн в нелинейной оптике и она дает обычно хорошее качественное объяснение основных черт явления, ее часто оказывается недостаточно 
для количественной трактовки возникающих ДУВ, а иногда даже малые поправки к НУШ, вызванные учетом малых дополнительных эффектов, приводят при достаточно долгой эволюции к радикальным отклонениям от предсказаний теории НУШ. Однако выход за рамки теории НУШ создает большие трудности для теории из-за того, что НУШ принадлежит к особому классу так называемых полностью интегрируемых уравнений, что было продемонстрировано в [13], и выход за рамки этого класса делает неприменимым метод обратной задачи рассеяния, используемый в теории НУШ. Например, оптические ударные волны наблюдались в интенсивных световых пучках, распространяющихся в фоторефрактивных кристаллах с дефокусирующей нелинейностью [14] с насыщением, так что при повышении интенсивности волны относительная роль нелинейности падает, что не учитывается теорией НУШ. В этом случае модуляционные уравнения Уизема оказываются слишком сложными для их аналитической трактовки в полном объеме. Однако для частного случая начального условия в виде скачка интенсивности метод работы [15] дает основные характеристики ДУВ [16]. Аналогичная теория может быть развита для оптических ДУВ в коллоидных средах [17]. Более общая форма начальных импульсов может быть рассмотрена с помощью недавно развитого метода [18].

Изменение вида нелинейности может приводить не только к количественным отличиям от теории НУШ, но и к качественно новым эффектам. Например, запаздывание нелинейного отклика среды на поле волны превращает НУШ в так называемое „нелинейное уравнение Шрёдингера с производной“, содержащее дополнительно к нелинейности керровского типа ее производную по времени. Такая модификация НУШ радикально изменяет теорию ДУВ, так что становятся возможными новые волновые структуры комбинированного типа, объединяющие, например, область осцилляций с волнами разрежения, а также другие волновые конфигурации [19].

К другим качественно новым эффектам приводят малые возмущения диссипативного типа, которые при достаточно больших временах становятся сравнимыми с малой модуляцией волнового пакета, что может приводить к стабилизации ДУВ, так что она приобретает стационарный профиль. Именно с таким явлением мы сталкиваемся при учете рамановского эффекта в распространении световых импульсов в световоде. Как было указано в работе [20], в малоамплитудном приближении НУШ с дополнительным рамановским членом может быть сведено к уравнению Кортевега-де Фриза-Бюргерса (КдФ-Б), для которого наличие стационарных ДУВ было установлено в работе [21] прямым расчетом в теории возмущений и в работах [22,23] с помощью метода Уизема (см. также [24]). Однако учет возмущающих членов такого типа в теории НУШ существенно сложнее $[25,26]$, и поэтому требует отдельного рассмотрения. Целью настоящей работы является изучить влияние рамановского эффекта, вызванного запаздывающим нели- нейным откликом системы, на динамику световых импульсов, распространяющихся в одномодовом волокне. Сначала в разд. 3 рассмотрим действие этого эффекта на динамику линейных волн, распространяющихся по однородному фону. Поскольку некоторые качественные черты поведения огибающей светового импульса в волокне выясняются уже из малоамплитудного предела уравнений эволюции, то в разд. 4 найдем ведущие дисперсионные и нелинейные поправки к бездисперсионному линейному распространению возмущений. Затем перейдем к описанию нескольких стадий развития ДУВ после опрокидывания волнового импульса. Как будет показано, на первом этапе, когда длина распространения света по волокну достаточно мала, рамановским эффектом можно пренебречь. Тогда система может описываться обычным НУШ с керровской нелинейностью, решение уравнений Уизема для которого в некоторых характерных случаях хорошо известно. На следующей стадии рамановский эффект вступает в силу, и эволюция ДУВ описывается возмущенными уравнениям Уизема, которые будут выведены в разд. 5 в рамках теории, развитой в работе [25]. Покажем, что этот эффект влияет по-разному на волны, распространяющиеся в различных направлениях. Наконец, будет показано, что ударная волна, направленная в положительном направлении оси времени $t$, приобретает стационарный характер, тогда как параметры ДУВ, распространяющейся в противоположную сторону, продолжают эволюционировать с увеличением амплитуды ДУВ и продолжительности волновой структуры. Полученные в работе аналитические результаты подтверждены численными расчетами.

\section{2. Основные уравнения}

Мы исходим из стандартного подхода [27], в котором динамика огибающей электрического поля $E(x, t)$ световой волны описывается НУШ с учетом нормальной дисперсии и дефокусирующей керровской нелинейности, тогда как затуханием пренебрегаем:

$$
\frac{\partial E}{\partial X}+\beta_{1} \frac{\partial E}{\partial T}+\frac{i}{2} \beta_{2} \frac{\partial^{2} E}{\partial T^{2}}-i \tilde{\gamma}|E|^{2} E=0,
$$

где $X-$ координата вдоль волновода, $T-$ время, $\beta_{1}$ - обратная групповая скорость волны $\left(v_{g r}=1 / \beta_{1}\right)$, $\beta_{2}$ - параметр, определяющий уширение импульса, $\tilde{\gamma}-$ нелинейный коэффициент, определяющийся для несущей частоты $\omega_{0}$ выражением

$$
\tilde{\gamma}=\frac{n_{2} \omega_{0}}{c_{l} E_{e f f}}, \quad E_{e f}=\pi w^{2} .
$$

Здесь $w$ - параметр гауссовой моды, $n_{2}$ - нелинейный показатель преломления, $c_{l}$ - скорость света в вакууме. Таким образом, второе слагаемое в уравнении эволюции (1) описывает перенос волны с групповой 
скоростью, а последний член отвечает керровской нелинейности. Слагаемое с коэффициентом $\beta_{2}>0$ является квадратичной дисперсией. Уравнение (1) заменой

$$
x=-\tilde{\gamma} I_{0} X, \quad t=\sqrt{\frac{\tilde{\gamma} I_{0}}{\beta_{2}}}\left(T-\beta_{1} X\right), \quad q=\frac{E}{\sqrt{I_{0}}},
$$

где $I_{0}-$ характерная интенсивность системы, преобразуется к обычному безразмерному виду

$$
i q_{x}+\frac{1}{2} q_{t t}-|q|^{2} q=0 .
$$

Как указано во Введении, на распространение импульсов вдоль достаточно длинных волокон существенно влияют малые эффекты, не учитываемые в приближении НУШ, такие как дисперсия более высокого порядка, самоукручение и комбинационное рассеяние (эффект Рамана). Влияние самоукручения на эволюцию ДУВ было подробно рассмотрено в работе [19], где было показано, что оно приводит к образованию сложных составных структур. Рамановский эффект в свою очередь описывает смешивание частоты вынужденного комбинационного саморассеяния, и его учет приводит к дополнительному члену в уравнении эволюции, так что уравнение для огибающей светового импульса в безразмерных переменных будет иметь вид

$$
i q_{x}+\frac{1}{2} q_{t t}-|q|^{2} q+\gamma q\left(|q|^{2}\right)_{t}=0 .
$$

Константа $\gamma$, характеризующая наклон линии ВКРусиления, обычно является малым параметром системы, что позволяет рассматривать последний член уравнения в качестве возмущения для описании ДУВ в теории Уизема. Стоит отметить, что самоукручение и рамановский эффект имеют различный характер, поэтому влияние каждого из них на систему можно изучать по отдельности.

Начнем исследования влияния рамановского члена с рассмотрения его действия на линейные волны, распространяющиеся по однородному фону.

\section{3. Линейные волны}

Пусть световой импульс распространяется вдоль однородного волнового фона $\mathrm{c}$ амплитудой $\sqrt{I_{0}}=$ $=\left|q_{0}\right|=$ const. Чтобы увидеть роль рамановского члена, найдем решение линеаризованного НУШ с рамановским членом для импульса в линейном приближении. В данном случае удобно перейти к переменной $\tilde{q}$, определяющейся заменой $q(x, t)=\tilde{q}(x, t) \exp \left(-i I_{0} x\right)$. Тогда уравнение (3) перепишется в виде

$$
i \tilde{q}_{x}+\frac{1}{2} \tilde{q}_{t t}+\left(I_{0}-|\tilde{q}|^{2}\right) \tilde{q}+\gamma \tilde{q}\left(|\tilde{q}|^{2}\right)_{t}=0 .
$$

Такая замена не меняет свойств уравнения, так как фаза волны определена с точностью до константы. Эволюция малого возмущения $\delta q$ на однородном фоне,

$$
\tilde{q}=\sqrt{I_{0}}+\delta q, \quad|\delta q| \ll \sqrt{I_{0}},
$$

может быть описана линеаризованным уравнением

$$
i \delta q_{x}+\frac{1}{2} \delta q_{t t}-I_{0}\left(\delta q+\delta q^{*}\right)+\gamma I_{0}\left(\delta q+\delta q^{*}\right)_{t}=0
$$

с начальным условием $\left.\delta q\right|_{x=0}=\delta q_{0}(t)$. После разделения действительной и мнимой частей,

$$
\delta q=A+i B,
$$

из (6) получим

$$
A_{x}+\frac{1}{2} B_{t t}=0, \quad B_{x}-\frac{1}{2} A_{t t}+2 I_{0} A-2 \gamma I_{0} A_{t}=0 .
$$

Функция $B$ может быть исключена, и тогда из этой системы следует линейное уравнение для $A$ :

$$
A_{x x}-I_{0} A_{t t}+\gamma I_{0} A_{t t t}+\frac{1}{4} A_{t t t t}=0 .
$$

Это уравнение можно решить методом Фурье. Для этого запишем закон дисперсии, которому удовлетворяют линейные гармонические волны $A \propto \exp [i(k x-\omega t)]$,

$$
k= \pm k(\omega), \quad k(\omega)=\omega \sqrt{\frac{\omega^{4}}{4}+I_{0}(1+i \gamma \omega)} .
$$

Мнимая единица в законе дисперсии означает, что при $\gamma \neq 0$ имеется затухание или усиление линейных волн. Общее решение уравнения (9) запишется в виде

$$
\begin{aligned}
A(x, t)= & \int_{-\infty}^{+\infty} W_{1}(\omega) e^{i(k(\omega) x-\omega t)} \frac{d \omega}{2 \pi} \\
& +\int_{-\infty}^{+\infty} W_{2}(\omega) e^{i(-k(\omega) x-\omega t)} \frac{d \omega}{2 \pi},
\end{aligned}
$$

где функции $W_{1,2}(\omega)$ определяются из начальных условий

$$
\begin{aligned}
A(0, t) & =\int_{-\infty}^{+\infty}\left[W_{1}(\omega)+W_{2}(\omega)\right] e^{-i \omega t} \frac{d \omega}{2 \pi}, \\
A_{x}(0, t) & =i \int_{-\infty}^{+\infty} k(\omega)\left[W_{1}(\omega)-W_{2}(\omega)\right] e^{-i \omega t} \frac{d \omega}{2 \pi} .
\end{aligned}
$$

После простых вычислений находим функции $W_{1,2}(\omega)$, выраженные через преобразование Фурье $\widehat{I_{0}^{\prime}}(\omega)$ и $\widehat{\varphi_{0}^{\prime}}(\omega)$ для начального (входного) возмущения интенсивности: $I^{\prime}(x, t)=2 \sqrt{I_{0}} A(x, t)$ и фазы $\varphi^{\prime}(x, t)=B(x, t) / \sqrt{I_{0}}$,

$$
\begin{aligned}
& W_{1}(\omega)=\frac{1}{4 \sqrt{I_{0}}}\left[\widehat{I_{0}^{\prime}}(\omega)-i \frac{\omega^{2}}{k(\omega)} I_{0} \widehat{\varphi_{0}^{\prime}}(\omega)\right], \\
& W_{1}(\omega)=\frac{1}{4 \sqrt{I_{0}}}\left[\widehat{I_{0}^{\prime}}(\omega)+i \frac{\omega^{2}}{k(\omega)} I_{0} \widehat{\varphi_{0}^{\prime}}(\omega)\right] .
\end{aligned}
$$

Пусть в начальный момент времени фаза волны посто- 


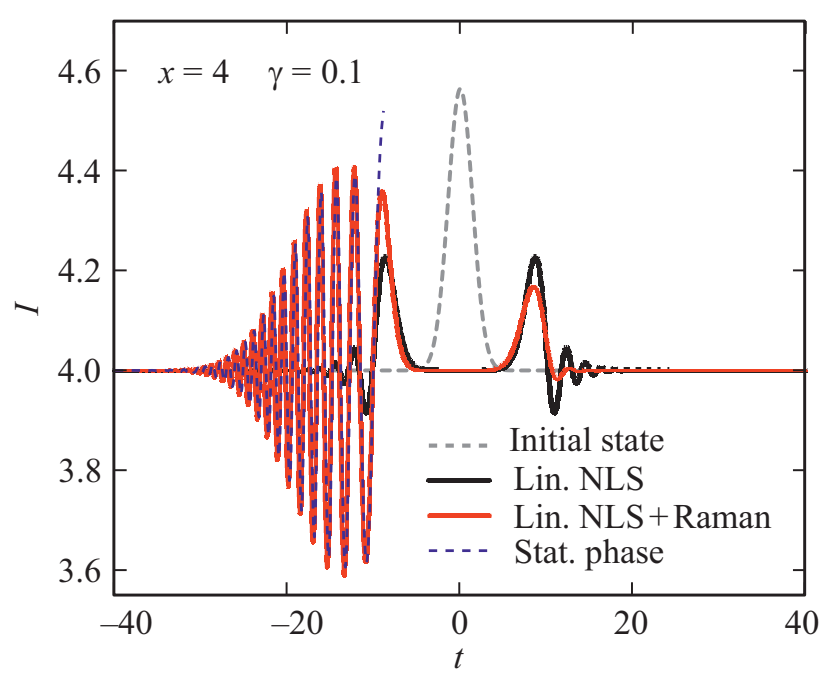

Рис. 1. Эволюция импульса в линейном приближении уравнения НУШ с рамановским членом (3) с $\gamma=0.1$. Штриховая серая линия показывает начальное возмущение, штриховая синяя линия соответствует методу седловой точки (16) и (17), красная непрерывная линия соответствует численному расчету интеграла (14) для $x=4$ с начальным возмущением (19) с $a=1$. Для сравнения черной сплошной линией показаны линейные волны, подчиняющиеся линеаризованному уравнению (6) с $\gamma=0$.

янна и имеется возмущение только интенсивности, т.е. $\varphi_{0}^{\prime}=0$, тогда

$$
I^{\prime}(t, x)=\frac{1}{4 \pi} \int_{-\infty}^{+\infty} \widehat{I_{0}^{\prime}}(\omega)\left[e^{i x f_{1}(\omega)}+e^{i x f_{2}(\omega)}\right] d \omega,
$$

где

$$
f_{1}(\omega)=k(\omega)-\omega \frac{t}{x}, \quad f_{2}(\omega)=-k(\omega)-\omega \frac{t}{x} .
$$

Этот интеграл можно оценить для большого расстояния распространения $x$ стандартным методом седловой точки, что приводит к

$$
\delta I(t, x) \simeq \frac{2 \widehat{I_{0}^{\prime}}\left(\omega_{0}^{(1)}\right)}{\sqrt{2 x\left|\frac{d^{2} f_{1}}{d \omega^{2}}\right|_{\omega_{0}^{(1)}}}} \cos \left(x f_{1}\left(\omega_{0}^{(1)}\right)-\frac{\pi}{4}\right)
$$

для волны, распространяющейся в отрицательном направлении оси $t$, и

$$
\delta I(t, x) \simeq \frac{2 \widehat{I_{0}^{\prime}}\left(\omega_{0}^{(2)}\right)}{\sqrt{2 x\left|\frac{d^{2} f_{2}}{d \omega^{2}}\right|_{\omega_{0}^{(2)}}}} \cos \left(x f_{2}\left(\omega_{0}^{(2)}\right)-\frac{\pi}{4}\right)
$$

для линейной волны, распространяющейся вправо, где $\omega_{0}^{(1)}$ и $\omega_{0}^{(2)}-$ значения $\omega$ в седловых точках, которые определяются уравнениями

$$
\frac{d f_{1}}{d \omega}=0, \quad \frac{d f_{2}}{d \omega}=0 .
$$

На рис. 1 показано сравнение численного расчета интеграла (14) с его приближенной оценкой (16) и (17) для начального возмущения

$$
I_{0}^{\prime}(t)=\frac{1}{\sqrt{\pi} a} \exp \left(-\frac{t^{2}}{a^{2}}\right), \quad \widehat{I_{0}^{\prime}}(\omega)=\exp \left(-\frac{\omega^{2} a^{2}}{4}\right)
$$

Как видим, импульс расщепляется на два меньших импульса, однако, в отличие от случая обычного НУШ, они не являются симметричными импульсами, распространяющимися в противоположных направлениях. Теперь эти два импульса имеют разные профили. Видно, что импульс, распространяющийся в положительном направлении оси $t$, затухает, а распространяющийся в отрицательном направлении усиливается. Ясно, что этот эффект есть результат действия рамановского члена в уравнении (3). Приближенное решение (16), (17) показывает, что амплитуда пакета линейных волн, распространяющихся влево, монотонно растет. Однако не стоит забывать, что данная теория справедлива для малых отклонений от фонового значения интенсивности. Следует также отметить, что асимптотическое решение (16) и (17) хорошо описывает волновой пакет даже при небольших длинах световода $x$.

На рис. 2 показана зависимость интенсивности линейных волн от времени, где в качестве начального возмущения выбран разрыв, который моделируем формулой

$$
I_{0}^{\prime}(t)=\frac{1}{2}\left\{I^{R}+I^{L}+\left(I^{R}-I^{L}\right) \tanh \left(\frac{t}{\Delta}\right)\right\},
$$

где „ширину разрыва“ $\Delta$ необходимо брать достаточно малой, чтобы ее влияние не сказывалось при достаточно

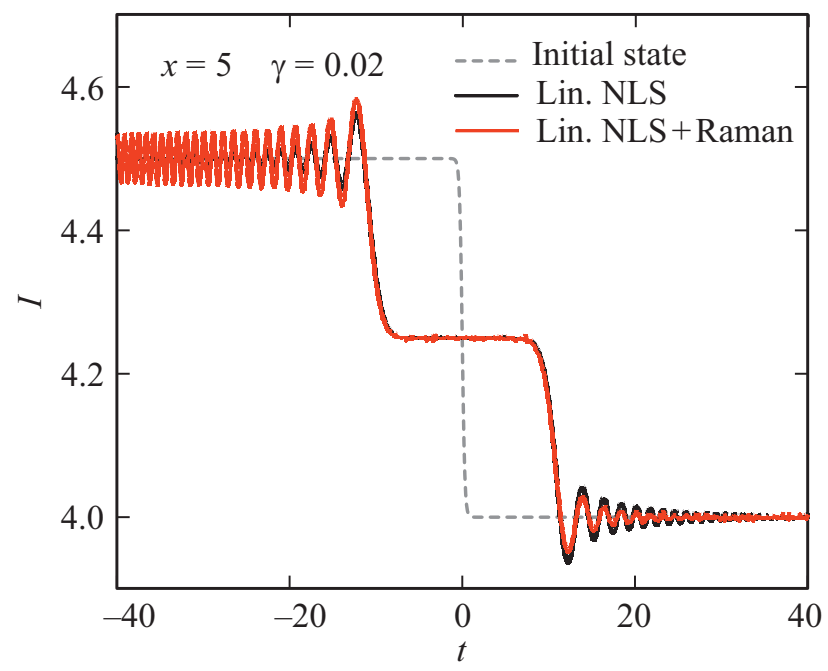

Рис. 2. Эволюция импульса в линейном приближении уравнения НУШ с рамановским членом (3) с $\gamma=0.02$. Штриховая серая линия показывает начальное возмущение в виде разрыва и красная непрерывная линия соответствует численному расчету интеграла (14) для $x=5$ с начальным возмущением (20) с $I^{L}=0.5, I^{R}=0$ и $\Delta=0.3$. Для сравнения черной сплошной линией показаны линейные волны, подчиняющиеся линеаризованному уравнению (6) с $\gamma=0$. 
больших временах. Константы $I^{R}$ и $I^{L}$ отвечают за граничные значения интенсивности света с правой и левой сторон от разрыва соответственно. Задача об эволюции начального разрыва является одной из фундаментальных в теории ДУВ, и мы детально рассмотрим ее в дальнейшем. Здесь же отметим, что разница между линейными волнами при $\gamma=0$ и при $\gamma \neq 0$ заметна даже при малых значениях $\gamma$, причем особенно для левой волны.

Обратимся теперь к исследованию влияния малых дисперсии и нелинейности на эволюцию импульса света.

\section{4. Предел малой амплитуды и слабой дисперсии}

Перейдем к исследованию влияния рамановского эффекта на эволюцию ДУВ в пределе малых амплитуд и дисперсии, когда они учитываются в главном приближении, т.е. нас интересуют ведущие дисперсионные и нелинейные поправки к бездисперсионному линейному распространению возмущений вдоль фонового импульса $I_{0}$ при учете рамановского члена в (3). Для исследования этого удобно пользоваться в качестве зависимых переменных функциями интенсивности $I(x, t)$ и модуляции частоты $u(x, t)$ (чирпа). Чтобы перейти к уравнениям для этих переменных, воспользуемся преобразованием Маделунга:

$$
q(x, t)=\sqrt{I(x, t)} \exp \left(i \int^{t} u\left(x, t^{\prime}\right) d t^{\prime}\right),
$$

после подстановки которого в уравнение (3), разделения действительной и мнимой частей и дифференцирования одного из уравнений получим систему

$$
\begin{aligned}
& I_{x}+(u I)_{t}=0 \\
& u_{x}+u u_{t}+I_{t}+\left(\frac{I_{t}^{2}}{8 I^{2}}-\frac{I_{t t}}{4 I}\right)_{t}=\gamma I_{t t} .
\end{aligned}
$$

Последнее слагаемое в левой части второго уравнения описывает эффекты дисперсии, а член, стоящий справа в этом уравнении, напоминает хорошо известную вязкость Бюргерса в теории волн на воде и других непрерывных средах $[8]$.

Пользуясь последней системой и применяя стандартную теорию возмущений по амплитуде возмущения и по слабой дисперсии [28], можно получить малоамплитудный аналог уравнения (3). Пусть волна распространяется в положительном направлении оси $t$, тогда в качестве приближенного уравнения для $I^{\prime}=I-I_{0}$ получим

$$
\begin{aligned}
\frac{\partial I^{\prime}}{\partial x}+\sqrt{I_{0}} \frac{\partial I^{\prime}}{\partial t} & +\frac{3}{2 \sqrt{I_{0}}} I^{\prime} \frac{\partial I^{\prime}}{\partial t} \\
& -\frac{1}{8 \sqrt{I_{0}}} \frac{\partial^{3} I^{\prime}}{\partial t^{3}}=\frac{1}{2} \gamma \sqrt{I_{0}} \frac{\partial^{2} I^{\prime}}{\partial t^{2}} .
\end{aligned}
$$

Это уравнение КдФ-Б. Как известно [22,24], эволюция волн, подчиняющихся данному уравнению, разбивается на три этапа. На первой стадии, когда $x \ll \gamma^{-1}$, член, характеризующий малую вязкость, слабо влияет на эволюцию ДУВ, и поэтому им пренебрегают. Затем на втором этапе, когда $x \sim \gamma^{-1}$, влияние вязкости становится сравнимым с модуляцией ДУВ. В этом случае, очевидно, вязкостью пренебречь нельзя, и тогда для описания ДУВ в силу малости коэффициента вязкости пользуются теорией возмущений. Наконец, на третьем этапе при $x \gg \gamma^{-1}$ ДУВ становится стационарной по $x$, т.е. профиль выходного сигнала перестает зависеть от длины световода. Подобного поведения ДУВ стоит ожидать и в случае приближения (23) для волны, распространяющейся влево.

Однако ситуация меняется для волн, распространяющихся в противоположном направлении. Для возмущения интенсивности $I^{\prime}$ предельное уравнение запишется теперь в виде

$$
\begin{aligned}
\frac{\partial I^{\prime}}{\partial x}-\sqrt{I_{0}} \frac{\partial I^{\prime}}{\partial t} & +\frac{1}{\sqrt{I_{0}}} I^{\prime} \frac{\partial I^{\prime}}{\partial t} \\
& +\frac{1}{8 \sqrt{I_{0}}} \frac{\partial^{3} I^{\prime}}{\partial t^{3}}=-\frac{1}{2} \gamma \sqrt{I_{0}} \frac{\partial^{2} I^{\prime}}{\partial t^{2}} .
\end{aligned}
$$

Это также уравнение КдФ-Б, однако стоит отметить, что здесь стоят иные знаки перед дисперсией и вязкостью. Это означает, что характер ДУВ изменится, и вместо стандартного затухания волны ожидается обратный процесс - усиление волны.

Такая разница между волнами, направленными в различные стороны, вызвана отсутствием симметрии уравнения (3) относительно замены $t \rightarrow-t$. Очевидно, что это свойство будет влиять на эволюцию импульса, описываемого полным уравнением (3). Обратимся к описанию формирования ДУВ и выводу уравнений, описывающих их динамику.

\section{5. Формирование ударной волны}

Сначала обсудим бездисперсионный предел гидродинамических уравнений, следующих из НУШ в пренебрежении дисперсией. В этом пределе уравнения (22) без члена, отражающего влияние рамановского эффекта, перейдут в систему

$$
\begin{aligned}
& I_{x}+(u I)_{t}=0, \\
& u_{x}+u u_{t}+I_{t}=0 .
\end{aligned}
$$

Первое уравнение этой системы можно интерпретировать как уравнение неразрывности для интенсивности $I(x, t)$, а второе - как уравнение Эйлера для „скорости течения“ $u(x, t)$. Эта система может быть приведена стандартным способом к диагональной форме Римана

$$
\frac{\partial r_{ \pm}}{\partial x}+\frac{1}{v_{ \pm}} \frac{\partial r_{ \pm}}{\partial t}=0
$$

для римановых инвариантов

$$
r_{ \pm}=\frac{u}{2} \pm \sqrt{I}
$$


с обратными скоростями

$$
\frac{1}{v_{ \pm}}=u \pm \sqrt{I}
$$

Введенные здесь функции понадобятся для выбора начального состояния, соответствующего волне, распространяющейся в определенном направлении.

Пусть начальные условия на входе в световод имеют ступенчатую форму:

$$
\begin{aligned}
& I(x=0)= \begin{cases}I^{L}, & t<0, \\
I^{R}, & t>0,\end{cases} \\
& u(x=0)= \begin{cases}u^{L}, & t<0, \\
u^{R}, & t>0 .\end{cases}
\end{aligned}
$$

При таком начальном условии опрокидывание волны будет происходить в момент $x=0$. Если бы эволюция огибающей света $q(x, t)$ описывалась уравнением (2), то в процессе распространения образовывались бы две характерные структуры: волна разрежения и ДУВ. Из вида гидродинамических уравнений (22) и малоамплитудного предела ясно, что в общем случае будет происходить формирование тех же структур, однако рамановский эффект будет влиять только на эволюцию ДУВ аналогично вязкости в случае динамики волн на воде, описываемых уравнением КдФ-Б. Тогда для рассмотрения динамики ударных волн имеет смысл ограничиться такими начальными условиями, эволюция которых будет приводить только к образованию ДУВ без образования волны разрежения, что существенно упрощает исследование. На языке введенных нами римановых инвариантов это будет означать, что начальное состояние должно выбираться согласно условиям $(a) r_{+}^{L}=r_{+}^{R}, r_{-}^{L}>r_{-}^{R}$ или (б) $r_{+}^{L}>r_{+}^{R}, r_{-}^{L}=r_{-}^{R}$, где верхний индекс обозначает соответствующую границу разрыва.

Определив таким образом начальные условия, перейдем к описанию трех стадий формирования ДУВ с учетом рамановского эффекта.

\section{1. Первый этап: $x \ll \gamma^{-1}$}

Так как опрокидывание происходит при $x=0$, то на этой стадии, когда $x \ll \gamma^{-1}$, должна быть учтена дисперсия. Однако модуляция волны относительно велика, и рамановским эффектом пока можно пренебречь. Тогда динамика описывается обычным НУШ (2), периодическое решение которого хорошо известно и может быть записано в форме [28]

$$
\begin{aligned}
I & =\frac{1}{4}\left(\lambda_{4}-\lambda_{3}-\lambda_{2}+\lambda_{1}\right)^{2}+ \\
& +\left(\lambda_{4}-\lambda_{3}\right)\left(\lambda_{2}-\lambda_{1}\right) \times \\
& \times \operatorname{sn}^{2}\left(\sqrt{\left(\lambda_{4}-\lambda_{2}\right)\left(\lambda_{3}-\lambda_{1}\right)} \theta, m\right), \\
u & =V-\frac{j}{I},
\end{aligned}
$$

где

$$
\begin{aligned}
& \theta=t-\frac{x}{V}, \quad \frac{1}{V}=\frac{1}{2} \sum_{i=1}^{4} \lambda_{i}, \\
& m=\frac{\left(\lambda_{2}-\lambda_{1}\right)\left(\lambda_{4}-\lambda_{3}\right)}{\left(\lambda_{4}-\lambda_{2}\right)\left(\lambda_{3}-\lambda_{1}\right)}, \quad 0 \leq m \leq 1 ; \\
& j=\frac{1}{8}\left(-\lambda_{1}-\lambda_{2}+\lambda_{3}+\lambda_{4}\right) \times \\
& \times\left(-\lambda_{1}+\lambda_{2}-\lambda_{3}+\lambda_{4}\right)\left(\lambda_{1}-\lambda_{2}-\lambda_{3}+\lambda_{4}\right) ;
\end{aligned}
$$

а действительные параметры $\lambda_{i}$ являются нулями полинома

$$
P(\lambda)=\prod_{i=1}^{4}\left(\lambda-\lambda_{i}\right)=\lambda^{4}-s_{1} \lambda^{3}+s_{2} \lambda^{2}-s_{3} \lambda+s_{4},
$$

где

$$
\begin{aligned}
& s_{1}=\sum_{i} \lambda_{i}, \quad s_{2}=\sum_{i<j} \lambda_{i} \lambda_{j}, \quad s_{3}=\sum_{i<j<k} \lambda_{i} \lambda_{j} \lambda_{k}, \\
& s_{4}=\lambda_{1} \lambda_{2} \lambda_{3} \lambda_{4},
\end{aligned}
$$

и $\lambda_{i}$ упорядочены согласно неравенствам

$$
\lambda_{1} \leq \lambda_{2} \leq \lambda_{3} \leq \lambda_{4}
$$

Как видим, через эти параметры выражаются обратная фазовая скорость волны $V^{-1}$, ее амплитуда $a=\left(\lambda_{4}-\lambda_{3}\right)\left(\lambda_{2}-\lambda_{1}\right)$, фоновая интенсивность $I_{0}=$ $=\left(\lambda_{4}-\lambda_{3}-\lambda_{2}+\lambda_{1}\right)^{2} / 4$, по которой распространяется волна, а также ее длина

$$
T=\frac{2 K(m)}{\sqrt{\left(\lambda_{1}-\lambda_{3}\right)\left(\lambda_{2}-\lambda_{4}\right)}},
$$

где $K(m)$ - полный эллиптический интеграл первого рода. Эти параметры являются в ДУВ медленными функциями от $x$ и $t$. Запись периодического решения в виде (30) обладает тем преимуществом, что параметры $\lambda_{i}$ являются римановыми инвариантами модуляционных уравнений и их эволюция определяется уравнениями Уизема в диагональной римановой форме [31,32]:

$$
\frac{\partial \lambda_{i}}{\partial x}+\frac{1}{v_{i}\left(\lambda_{1}, \lambda_{2}, \lambda_{3}, \lambda_{4}\right)} \frac{\partial \lambda_{i}}{\partial t}=0, \quad i=1,2,3,4 .
$$

Здесь $v_{i}$ суть характеристические скорости Уизема:

$$
\begin{aligned}
& \frac{1}{v_{1}}=\frac{1}{2} \sum_{i=1}^{4} \lambda_{i}-\frac{\left(\lambda_{4}-\lambda_{1}\right)\left(\lambda_{2}-\lambda_{1}\right) K}{\left(\lambda_{4}-\lambda_{1}\right) K-\left(\lambda_{4}-\lambda_{2}\right) E}, \\
& \frac{1}{v_{2}}=\frac{1}{2} \sum_{i=1}^{4} \lambda_{i}+\frac{\left(\lambda_{3}-\lambda_{2}\right)\left(\lambda_{2}-\lambda_{1}\right) K}{\left(\lambda_{3}-\lambda_{2}\right) K-\left(\lambda_{3}-\lambda_{1}\right) E}, \\
& \frac{1}{v_{3}}=\frac{1}{2} \sum_{i=1}^{4} \lambda_{i}-\frac{\left(\lambda_{4}-\lambda_{3}\right)\left(\lambda_{3}-\lambda_{2}\right) K}{\left(\lambda_{3}-\lambda_{2}\right) K-\left(\lambda_{4}-\lambda_{2}\right) E}, \\
& \frac{1}{v_{4}}=\frac{1}{2} \sum_{i=1}^{4} \lambda_{i}+\frac{\left(\lambda_{4}-\lambda_{3}\right)\left(\lambda_{4}-\lambda_{1}\right) K}{\left(\lambda_{4}-\lambda_{1}\right) K-\left(\lambda_{3}-\lambda_{1}\right) E},
\end{aligned}
$$


где $E=E(m)$ - эллиптический интеграл второго рода.

В пределе $m \rightarrow 1\left(\lambda_{3} \rightarrow \lambda_{2}\right)$ бегущая волна трансформируется в солитонное решение на фоне постоянной плотности:

$$
\begin{aligned}
& I=\frac{1}{4}\left(\lambda_{4}-\lambda_{1}\right)^{2}-\frac{\left(\lambda_{4}-\lambda_{2}\right)\left(\lambda_{2}-\lambda_{1}\right)}{\operatorname{ch}^{2}\left(\sqrt{\left(\lambda_{4}-\lambda_{2}\right)\left(\lambda_{2}-\lambda_{1}\right)} \theta\right)}, \\
& \theta=x-\frac{1}{2}\left(\lambda_{1}+2 \lambda_{2}+\lambda_{4}\right) t .
\end{aligned}
$$

В другом (малоамплитудном) пределе $m \rightarrow 0\left(\lambda_{3} \rightarrow \lambda_{4}\right.$ или $\left.\lambda_{2} \rightarrow \lambda_{1}\right)$ амплитуда волны стремится к нулю, а плотность обращается в фоновое значение. Существенно, что в этих пределах пара уиземовских скоростей переходит в римановы скорости бездисперсионного предела. Это означает, что края ДУВ сшиваются с плавными решениями гидродинамического приближения бездисперсионного предела, применимого для описания эволюции волны вне ДУВ.

Так как начальное условие не содержит параметров, имеющих размерность времени, то модуляционные параметры зависят только от автомодельной переменной $\tau=t / x$. Поэтому уравнения Уизема сводятся к

$$
\frac{d \lambda_{i}}{d \tau}\left(v_{i}-\tau\right)=0
$$

Отсюда следует, что лишь один инвариант Римана является переменным, а остальные три должны иметь постоянные значения. Таким образом, зная входные условия, можно получить вид ДУВ для $x \ll \gamma^{-1}$. Характерные ситуации были изучены в $[10,11]$, и мы не будем здесь останавливаться на деталях.

\section{2. Второй этап: $x \sim y^{-1}$}

На этой стадии, когда $x \sim y^{-1}$, эффекты, связанные с рамановским членом, начинают играть существенную роль и конкурировать с влиянием модуляции. Динамика ДУВ регулируется полным уравнением НУШ с рамановским членом (3). Локальная форма волны попрежнему описывается периодическим решением (30), однако уравнения Уизема теперь становятся неоднородными из-за появления возмущающего члена. При их выводе воспользуемся методом, развитым в работе [25]. Этот метод можно сформулировать следующим образом. Пусть уравнения для эволюции волновых переменных $\varphi_{m}(x, t)$ имеют вид

$$
\begin{gathered}
\varepsilon \frac{\partial \varphi_{m}}{\partial t}=K_{m}\left(\varphi_{n}, \varepsilon \frac{\partial \varphi_{m}}{\partial x}, \varepsilon^{2} \frac{\partial^{2} \varphi_{m}}{\partial x^{2}}, \ldots\right) \\
+R_{m}\left(\varphi_{n}, \varepsilon \frac{\partial \varphi_{m}}{\partial x}, \varepsilon^{2} \frac{\partial^{2} \varphi_{m}}{\partial x^{2}}, \ldots\right) \\
m, n=1, \ldots, N
\end{gathered}
$$

где $\varepsilon \ll 1$ - малый параметр, измеряющий дисперсионные эффекты. Функции $K_{m}$ соответствуют „ведущей“ интегрируемой части уравнений без учета возмущений, а функции $R_{m}$ являются возмущающими систему членами. Предполагается, что невозмущенная система может быть представлена как условие совместимости двух линейных уравнений

$$
\begin{aligned}
\varepsilon^{2} \chi_{x x} & =\mathscr{A} \chi \\
\chi_{t} & =-\frac{1}{2} \mathscr{B}_{x} \chi+\mathscr{B} \chi_{x},
\end{aligned}
$$

где $\mathscr{A}$ и $\mathscr{B}$ зависят от функций $\varphi_{n}$, их пространственных производных и спектрального параметра $\lambda$. Это условие выполняется для НУШ и позволяет использовать для его исследования мощный метод обратной задачи рассеяния [28]. Линейные уравнения второго порядка (40) имеют два базисных решения $\chi_{ \pm}$, а их произведение $g=\chi_{+} \chi_{-}$удовлетворяет дифференциальному уравнению третьего порядка, которое можно проинтегрировать один раз, чтобы получить

$$
\frac{\varepsilon^{2}}{2} g g_{x x}-\frac{\varepsilon^{2}}{4} g_{x}^{2}-\mathcal{A g}^{2}=\sigma P(\lambda),
$$

где коэффициент $\sigma$ определяется согласно выражению $\left.\mathcal{A}\right|_{\lambda \rightarrow \infty} \rightarrow-\sigma \lambda^{r}$, а $P(\lambda)$ - полином $\lambda$ степени $M$ с нулями, равными $\lambda_{i}$.

В модулированной волне параметры $\lambda_{i}$ становятся медленными функциями от $x$ и $t$, их эволюция описывается возмущенными уравнениями Уизема, которые в случае уравнения (39) запишутся в виде

$$
\begin{aligned}
& \frac{\partial \lambda_{i}}{\partial t}-\frac{\langle\mathscr{B} / g\rangle}{\langle 1 / g\rangle} \frac{\partial \lambda_{i}}{\partial x}= \\
&=\lim _{\varepsilon \rightarrow 0}\left\{\frac{\sigma}{\langle 1 / g\rangle \prod_{j \neq k}\left(\lambda_{k}-\lambda_{j}\right)} \times\right. \\
&\left.\times \sum_{m=1}^{N} \sum_{l=0}^{A_{m}}\left\langle\frac{\partial \mathscr{A}}{\partial \varphi_{m}^{(l)}} \frac{\partial^{l} R_{m}}{\partial x^{l}} g\right\rangle\right\}, \\
& i=1, \ldots, M,
\end{aligned}
$$

где $A_{m}$ обозначает высший порядок производной от функций $\varphi_{m}$, входящей в $\mathcal{A}$, а угловые скобки обозначают усреднение по одной длине волны $L$. Bсе $\lambda$ после усреднения берутся равными $\lambda_{i}$.

Применим эту теорию к НУШ с рамановским членом

$$
i \varepsilon q_{x}+\frac{\varepsilon^{2}}{2} q_{t t}-|q|^{2} q=-\varepsilon \mathcal{\gamma} q\left(|q|^{2}\right)_{t}
$$

Введенный здесь положительный малый параметр $\varepsilon \ll 1$ явным образом отражает малость дисперсионных эффектов, что предполагается в теории ДУВ, так что после усреднения следует оставлять лишь ведущее приближение по этому параметру. В уравнении (43) присутствуют две переменные функции $q$ и $q^{*}$ и соответственно два возмущающих члена:

$$
R_{q}=i \gamma q\left(|q|^{2}\right)_{t}, \quad R_{q^{*}}=-i \gamma q^{*}\left(|q|^{2}\right)_{t} .
$$


Для невозмущенного НУШ функции $\mathcal{A}$ и $\mathscr{B}$, входящие в линейные уравнения (40), имеют вид

$$
\begin{aligned}
& \mathscr{A}=-\lambda^{2}+i \varepsilon \lambda \frac{q_{t}}{q}+|q|^{2}-\frac{\varepsilon^{2}}{2} \frac{q_{t t}}{q}+\frac{3 \varepsilon^{2}}{4} \frac{q_{t}^{2}}{q^{2}}, \\
& \mathscr{B}=-\lambda+\frac{i \varepsilon}{2} \frac{q_{t}}{q} .
\end{aligned}
$$

Усреднение может быть выполнено с использованием уравнений, известных из теории периодических решений НУШ:

$$
\begin{aligned}
& g=\lambda-\mu, \quad \varepsilon \frac{d \mu}{d t}=2 \sqrt{-P(\mu)}, \\
& \frac{1}{V}=\frac{s_{1}}{2},
\end{aligned}
$$

где $P(\mu)$ и $s_{1}$ определяются выражениями (32) и (33). Для вычисления правых частей уравнений Уизема нам также понадобится выражение для параметра $\mu$ [28]

$$
\begin{aligned}
\mu(I) & =\frac{s_{1}}{4}+\frac{-j+i \sqrt{\mathscr{R}(I)}}{2 I}, \quad \varepsilon \frac{d I}{d t}=2 \sqrt{\mathscr{R}(I)}, \\
\mathscr{R}(I) & =\left(I-I_{1}\right)\left(I-I_{2}\right)\left(I-I_{3}\right),
\end{aligned}
$$

где $I_{i}-$ нули функции $\mathscr{R}(I)$, связанные с римановыми инвариантами $\lambda_{i}$ посредством формул

$$
\begin{aligned}
& I_{1}=\frac{1}{4}\left(\lambda_{1}-\lambda_{2}-\lambda_{3}+\lambda_{4}\right)^{2}, \\
& I_{2}=\frac{1}{4}\left(\lambda_{1}-\lambda_{2}+\lambda_{3}-\lambda_{4}\right)^{2}, \\
& I_{3}=\frac{1}{4}\left(\lambda_{1}+\lambda_{2}-\lambda_{3}-\lambda_{4}\right)^{2},
\end{aligned}
$$

а $j$ дано уравнением (31). В результате получим уравнения Уизема для римановых инвариантов $\lambda_{i}$ в виде

$$
\begin{aligned}
\frac{\partial \lambda_{i}}{\partial x}+ & \frac{1}{v_{i}} \frac{\partial \lambda_{i}}{\partial t}= \\
= & \frac{\gamma j}{\partial T / \partial \lambda_{i}} \frac{\lambda_{i}}{\prod_{j \neq i}\left(\lambda_{i}-\lambda_{j}\right)} \int_{I_{1}}^{I_{2}} \frac{\sqrt{\mathscr{R}(I)}}{I^{2}} d I, \\
i & =1,2,3,4,
\end{aligned}
$$

где длина волны $T$ выражается уравнением (34). Интеграл в правой части может быть выражен через эллиптические интегралы. Однако такое выражение будет очень сложным, и легче иметь дело с его исходной формой.

Решение возмущенных уравнений Уизема (49) определяет эволюцию параметров $\lambda_{i}$ из-за неравномерной модуляции волн и слабого эффекта типа вязкости, вызванного рамановским членом. Из рассмотренных ранее малоамплитудных пределов типа КдФ естественно ожидать, что ДУВ, распространяющиеся в положительном направлении, асимптотически будут стремиться к стационарным волнам, и некоторые их характеристики можно вычислить аналитически. Амплитуды ДУВ, распространяющиеся влево, будут непрерывно расти, и их решение можно найти численно из полученных возмущенных уравнений Уизема.

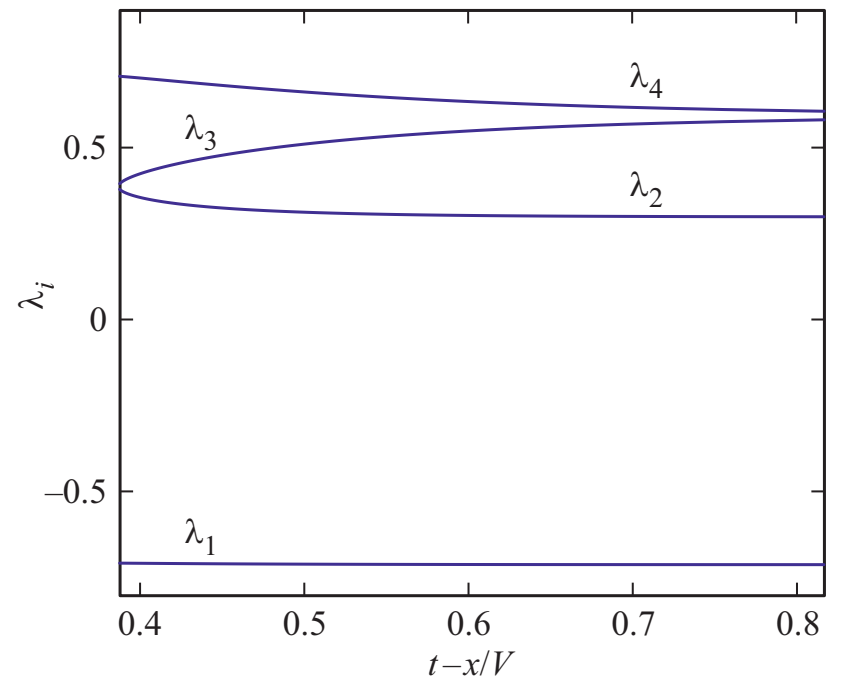

Рис. 3. Римановы инварианты $\lambda_{i}$ как функции от $\theta=t-x / V$.

\section{3. Третий этап: $x \gg y^{-1}$}

На расстояниях $x \gg \gamma^{-1}$ ДУВ, распространяющаяся в положительном направлении оси $t$, становится стационарной, так как для таких $x$ рамановский эффект уравновесит влияние модуляции волны. Это означает, что ДУВ движется как целое с постоянной скоростью $V$ и ее профиль не изменяется. Тогда ДУВ будут определяться возмущенными уравнениями Уизема (49) с параметрами $\lambda_{i}$, зависящими от $\theta=t-x / V$. Если предположить, что функция $s_{1}$ является интегралом уравнений Уизема, т. е. $s_{1}=$ const при условии $1 / V=s_{1} / 2$, то уравнения Уизема примут более простую форму:

$$
\frac{\partial \lambda_{i}}{\partial \theta}=\frac{\lambda_{i} Q}{\prod_{j \neq i}\left(\lambda_{i}-\lambda_{j}\right)}, \quad i=1,2,3,4,
$$

где

$$
Q=\frac{2 \gamma j}{T} \int_{I_{1}}^{I_{2}} \frac{\sqrt{\mathscr{R}(I)}}{I^{2}} d I
$$

Покажем, что наше предположение о постоянстве $s_{1}$ оправдано и структура этих уравнений фактически обеспечивает три интеграла $s_{1}, s_{2}$ и $s_{4}$. Это утверждение может быть доказано с использованием тождеств Якоби, которые вытекают из очевидного тождества

$$
\sum_{i=1}^{M} \frac{\prod_{j \neq i}\left(\lambda-\lambda_{j}\right)}{\prod_{j \neq i}\left(\lambda_{i}-\lambda_{j}\right)}=1,
$$

где в левой части имеем многочлен степени $M-1$, который равен единице в точках $\lambda=\lambda_{i}, i=1, \ldots, M$ и, следовательно, равен единице тождественно. В нашем 


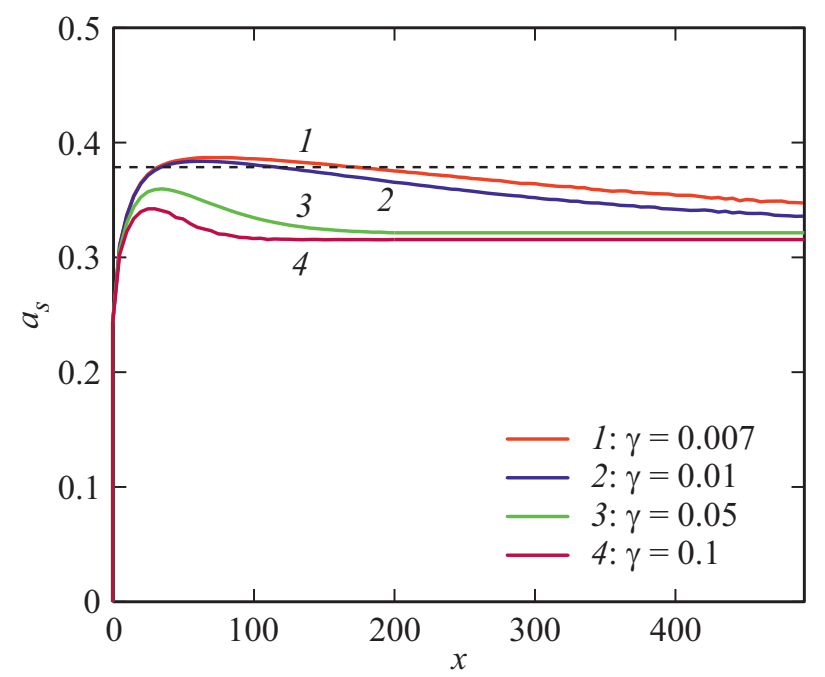

Рис. 4. Зависимость амплитуды ведущего солитона от длины распространения $x$ при различных значениях константы $\gamma$, характеризующих рамановский эффект. Аналитический результат, полученный с помощью теории Уизема, показан черной штриховой линией, а численные результаты, полученные решением уравнения (3), показаны сплошными кривыми.

случае $M=4$, так что из этого тождества получим

$$
\begin{aligned}
& \sum_{i=1}^{4} \frac{\lambda_{i}^{n}}{\prod_{j \neq i}\left(\lambda_{i}-\lambda_{j}\right)}= \begin{cases}0, & n=0,1,2, \\
1, & n=3,\end{cases} \\
& \sum_{i=1}^{4} \frac{\lambda_{i}^{n} \sum_{j}{ }^{\prime} \lambda_{j}}{\prod_{j \neq i}\left(\lambda_{i}-\lambda_{j}\right)}= \begin{cases}0, & n=0,1,3, \\
-1, & n=2,\end{cases} \\
& \sum_{i=1}^{4} \frac{\lambda_{i}^{n} \sum_{j, k}{ }^{\prime} \lambda_{j} \lambda_{k}}{\prod_{j \neq i}\left(\lambda_{i}-\lambda_{j}\right)}= \begin{cases}0, & n=0,2,3, \\
1, & n=1,\end{cases} \\
& \sum_{i=1}^{4} \frac{\lambda_{i}^{n} s_{4}}{\prod_{j \neq i}\left(\lambda_{i}-\lambda_{j}\right)}= \begin{cases}0, & n=0,1,2, \\
-1, & n=-1,\end{cases}
\end{aligned}
$$

где сумма со штрихом означает, что в ней все члены с множителем $\lambda_{i}$ опущены. Отсюда следует, что

$$
\frac{d s_{1}}{d \theta}=0, \quad \frac{d s_{2}}{d \theta}=0, \quad \frac{d s_{3}}{d \theta}=Q, \quad \frac{d s_{4}}{d \theta}=0,
$$

т.е. уравнения (50) имеют три интеграла движения $s_{1}=$ const, $s_{2}=$ const и $s_{4}=$ const. Таким образом, система уравнений (50) свелась к одному обыкновенному дифференциальному уравнению

$$
\frac{d s_{3}}{d \theta}=\frac{2 \gamma j}{T} \int_{I_{1}\left(s_{3}\right)}^{I_{2}\left(s_{3}\right)} \frac{\sqrt{\mathscr{R}(I)}}{I^{2}} d I
$$

с начальным условием $s_{3}\left(\theta_{0}\right)=s_{3}^{0}$, которое удобно брать на солитонном крае ДУВ.

Из условий сшивки на краях ДУВ находим, что на солитонном крае

$$
\lambda_{1}^{L}=r_{-}^{L}, \quad \lambda_{4}^{L}=r_{-}^{L} \quad \text { при } \quad \lambda_{3}^{L}=\lambda_{2}^{L},
$$

и на малоамплитудном краю

$$
\lambda_{1}^{R}=r_{-}^{R}, \quad \lambda_{2}^{R}=r_{+}^{R} \quad \text { при } \quad \lambda_{3}^{R}=\lambda_{4}^{R} .
$$

Диаграмма римановых инвариантов показана на рис. 3 . Так как в данном случае рассматриваем волну, распространяющуюся в положительном направлении, мы имеем $r_{+}^{L}>r_{+}^{R}, r_{-}^{L}=r_{-}^{R}$. Это означает, что $\lambda_{4}^{L}>\lambda_{2}^{R}$ и $\lambda_{1}^{L}=\lambda_{1}^{R}$. Тогда из постоянства функций $s_{1}$ и $s_{2}$ получим

$$
\begin{aligned}
\lambda_{2}^{L} & =\frac{1}{2}\left(\lambda_{2}^{R}+\sqrt{\lambda_{2}^{R} \lambda_{4}^{L}}\right) \\
& =\frac{1}{4}\left(u^{R}+2 \sqrt{I^{R}}+\sqrt{\left(u^{L}+2 \sqrt{I^{L}}\right)\left(u^{R}+2 \sqrt{I^{R}}\right)}\right)
\end{aligned}
$$

и

$$
\begin{aligned}
\lambda_{4}^{R} & =\frac{1}{2}\left(\lambda_{4}^{L}+\sqrt{\lambda_{2}^{R} \lambda_{4}^{L}}\right) \\
& =\frac{1}{4}\left(u^{L}+2 \sqrt{I^{L}}+\sqrt{\left(u^{L}+2 \sqrt{I^{L}}\right)\left(u^{R}+2 \sqrt{I^{R}}\right)}\right) .
\end{aligned}
$$

Таким образом, мы знаем все римановы инварианты на обоих краях ДУВ, т.е. нам известны интегралы $s_{1}$ и $s_{4}$ вдоль всей ударной волны. Отсюда можем получить скорость волны и амплитуду ведущего солитона:

$$
\begin{aligned}
\frac{1}{V}=\frac{s_{1}}{2} & =\frac{1}{2}\left(\lambda_{1}^{L}+\lambda_{4}^{L}+\lambda_{2}^{R}+\sqrt{\lambda_{2}^{R} \lambda_{4}^{L}}\right) \\
=\frac{1}{4} & \left(2 u^{L}+u^{R}+2 \sqrt{I^{R}}\right. \\
& \left.+\sqrt{\left(u^{L}+2 \sqrt{I^{L}}\right)\left(u^{R}+2 \sqrt{I^{R}}\right)}\right),
\end{aligned}
$$

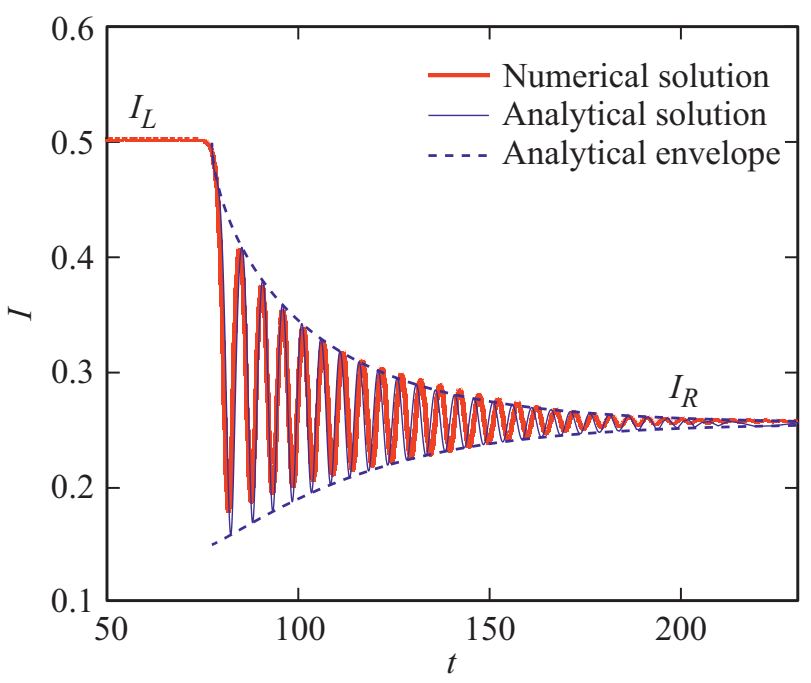

Рис. 5. Стационарная ДУВ. Показано сравнение теории Уизема (синий цвет) с численным решением уравнения (3) (красный цвет) для $\gamma=0.05$ с граничными условиями $I_{L}=0.5$, $I_{R}=0.257, u_{L}=0$ и $u_{R}=-0.4$ при $x=200$. Штриховыми линями показаны результаты теории Уизема для огибающих. 


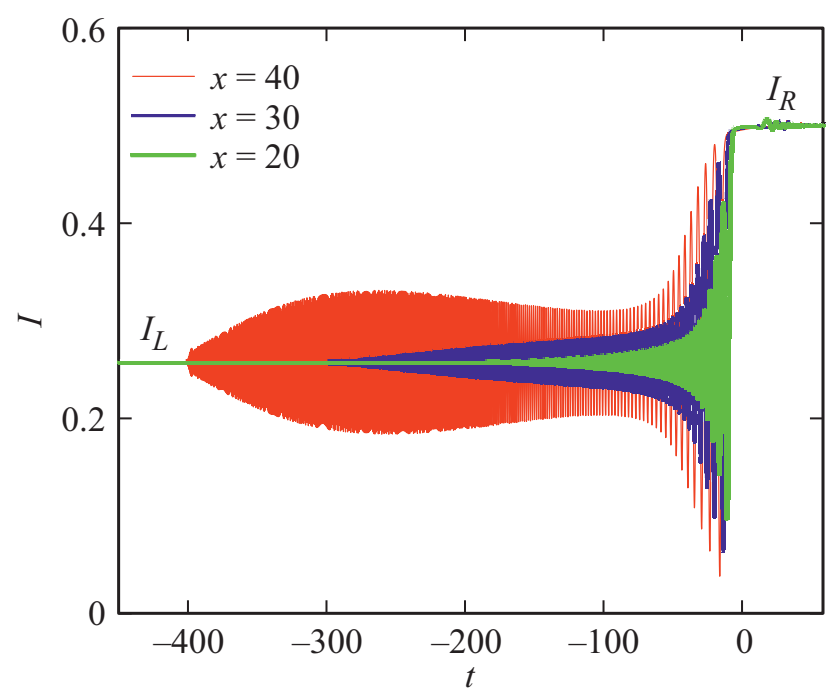

Рис. 6. ДУВ, распространяющаяся в отрицательном направлении оси $t$, для $\gamma=0.1$ с граничными условиями $I_{L}=0.257$, $I_{R}=0.5, u_{L}=0.4$ и $u_{R}=0$.

$$
\begin{aligned}
a_{s}=\frac{1}{4} & \left(2 \lambda_{4}^{L}-\lambda_{2}^{R}-\sqrt{\lambda_{2}^{R} \lambda_{4}^{L}}\right)\left(\lambda_{2}^{R}-2 \lambda_{1}^{L}+\sqrt{\lambda_{2}^{R} \lambda_{4}^{L}}\right) \\
=I^{L} & -\frac{1}{16}\left[2 \sqrt{I^{L}}-2 u^{L}+u^{R}\right. \\
& \left.+\sqrt{\left(u^{L}+2 \sqrt{I^{L}}\right)\left(u^{R}+2 \sqrt{I^{R}}\right)}\right]^{2} .
\end{aligned}
$$

Важно отметить, что скорость ДУВ и амплитуда солитона зависят только от начальных параметров и не зависят от постоянной $\gamma$, отражающей эффект Рамана. Численные результаты зависимости амплитуды солитона от координаты $x$ показаны на рис. 4. Как видим, имеется некоторое отклонение теории от численного расчета, вызванное, по-видимому, тем, что в теории Уизема не учитываются неадиабатические эффекты, которые могут играть существенную роль в ДУВ, описываемых неинтегрируемыми уравнениями, к которым относится НУШ с учетом рамановского члена. Такого рода отличия уже отмечались в работах $[16,29]$ для других физических систем. Несмотря на указанное отличие, в целом ДУВ достаточно хорошо описывается теорией Уизема, как это проиллюстрировано на рис. 5. Как видим, соответствие между результатами теории Уизема и численными расчетами заметно улучшается с уменьшением амплитуды волны.

Для волны, распространяющейся в противоположную сторону, будет обратная ситуация - амплитуда осцилляций в волне и ее протяженность будут непрерывно расти. Построение аналитической теории такой нестационарной нелинейной структуры весьма затруднительно и приходится обращаться к численному расчету. На рис. 6 показан пример такой волны, полученной численным решением уравнения (3). Видно, что профиль волны существенно изменяется с ростом расстояния $x$. При этом на достаточно больших длинах волновода $x$ можно отметить значительное увеличение амплитуды отделяющегося от ДУВ волнового пакета, описание которого подчиняется с хорошим приближением линейной теорией разд. 3.

\section{6. Заключение}

В работе развита аналитическая теория распространение достаточно длинных импульсов света в волокнах, описываемых НУШ, модифицированным малым слагаемым, характеризующим эффект Рамана. Рассмотрены основные этапы формирования ударной волны с начальным профилем в виде „ступеньки“ и построены аналитические решения для начальных нестационарных и конечных устойчивых стадий развития ударной волны с использованием метода Уизема для уравнений с возмущающими членами.

В принципе, найденные здесь результаты можно наблюдать экспериментально в системах, аналогичных тем, которые использовались в недавнем эксперименте [12]. Однако следует иметь в виду, что в стандартных волокнах помимо эффекта, связанного с комбинационным рассеянием, возникает также самоукручение. Однако проявления этих двух эффектов весьма различны, и поэтому их можно идентифицировать по отдельности. Как было показано в работе [19], основным следствием самоукручения является формирование комбинированных ударных волн, вызванных немонотонной зависимостью нелинейного члена от амплитуды волны, тогда как комбинационное рассеяние приводит к образованию стационарных ударных волн конечной длины. При этом эффект Рамана, как правило, намного сильнее, чем эффект самоукручения. Теория, разработанная в этой работе, показывает, что метод Уизема обеспечивает общий достаточно эффективный подход для описания ДУВ, наблюдаемых в световодах и других оптических системах (см., например, [30].

\section{Конфликт интересов}

Авторы заявляют, что у них нет конфликта интересов.

\section{Список литературы}

[1] Tomlinson W.J., Stolen R.H., Johnson A.M. // Optics Lett. 1985. V. 10. № 9. P. 457-459. doi 10.1364/OL.10.000457

[2] Rothenberg J.E., Grischkowsky D. // Phys. Rev. Lett. 1989. V. 62. N 5. P. 531. doi 10.1103/PhysRevLett.62.531

[3] Benjamin T.B. and Lighthill M.J. // Proc. R. Soc. London Ser. A. 1954. V. 224. N 1159. P. 448-460. doi 10.1098/rspa.1954.0172

[4] Taylor R.J., Baker D.R., and Ikezi H. // Phys. Rev. Lett. 1970. V. 24. N 5. P. 206. doi 10.1103/PhysRevLett.24.206

[5] Сагдеев P.3. Коллективные процессы и ударные волны в разреженной плазма, Вопросы теории плазмы, ред М.А. Леонтович, вып. 4, Москва, Атомиздат, 1964. 
[6] Гуревич А.В., Питаевский Л.П. // ЖЭТФ. 1973. Т. 65. C. 590 .

[7] Whitham G.B. // Proc. Roy. Soc. London, A. 1965. V. 283. N 1393. P. 238. doi 10.1098/rspa.1965.0019

[8] Уизем Джс. Линейные и нелинейные волны. М.: Мир, 1977; Whitham G. B. Linear and Nonlinear Waves. New York: Wiley Interscience. 1974.

[9] El G.A., Hoefer M.A. // Physica D. 2016. V. 333. P. 11-65. doi 10.1016/j.physd.2016.04.006

[10] Гуревич А.В., Крылов А.Л. // ЖЭТФ. 1987. Е. 92. С. 1684.

[11] El G.A., Geogjaev V.V., Gurevich A.V., Krylov A.L. // Physica D. 1995. V. 87. Issues 1-4. P. 186-192. doi 10.1016/01672789(95)00147-V

[12] Xu G., Conforti M., Kudlinski A., Mussot A., and Trillo S. // Phys. Rev. Lett. 2017. V. 118. N 254101. 10.1103/PhysRevLett.118.254101

[13] Захаров В.Е., Шабат А.В. // ЖЭТФ. 1971. Т. 61. № 1. C. $118-134$.

[14] Wan W., Jia S., and Fleischer J.W. // Nature Phys. 2007. V. 3. P. 46-51. doi $10.1038 /$ nphys 486

[15] El G.A. // Chaos. 2005. V. 15. N 037103. doi $10.1063 / 1.1947120$

[16] El G.A., Gammal A., Khamis E.G., Kraenkel R.A., Kamchatnov A.M. // Phys. Rev. A. 2007. V. 76. N 053813. doi 10.1103/PhysRevA.76.053813

[17] An X., Marchant T.R., Smyth N.F. // Physica D. 2017. V. 342. P. 45-56. doi 10.1016/j.physd.2016.11.004

[18] Kamchatnov A.M. // Phys. Rev. E. 2019. V. 99. N 012203. doi 10.1103/PhysRevE.99.012203

[19] Ivanov S.K., Kamchatnov A.M. // Phys. Rev. A. 2017. V. 96. N 053844. doi 10.1103/PhysRevA.96.053844

[20] Kivshar Y.S. // Phys. Rev. A. 1990. V. 42. 1757 N 3. P. 42. doi 10.1103/PhysRevA.42.1757; Kivshar Yu.S. and Malomed B.A. // Opt. Lett. 1993. V. 18. P. 485-487.

[21] Johnson R.S. // J. Fluid Mech. 1970. V. 42. P. 49-60. doi $10.1017 / \mathrm{S} 0022112070001064$

[22] Гуревич А.В. и Питаевский Л.П. // ЖЭТФ. 1987. Т. 93. C. 871.

[23] Авилов В.В., Кричевер И.М., Новиков С.П. // ДАН СССР. 1987. T. 295. C. 345.

[24] Kamchatnov A.M. // Physica D. 2016. V. 333. P. 99-106. doi 10.1016/j.physd.2015.11.010

[25] Kamchatnov A.M. // Physica D. 2004. V. 188. Issues 3-4. P. 247-261. doi 10.1016/j.physd.2003.07.008

[26] Larré P.-É., Pavloff N., Kamchatnov A.M. // Phys. Rev. B. 2012. V. 86. N 165304. doi 10.1103/PhysRevB.86.165304

[27] Кивщарь Ю.С., Агравал Г.П. Оптические солитоны. От волоконных световодов до фотонных кристаллов. М.: ФИЗМАТЛИТ, 2005; Kivshar Yu.S. and Agrawal G.P. Optical Solitons. From Fibers to Photonic Crystals. Amsterdam: Academic Press, 2003.

[28] Kamchatnov A.M. Nonlinear Periodic Waves and Their Modulations-An Introductory Course. Singapore: World Scientific, 2000.

[29] El G.A., Grimshaw R.H.J., Smyth N.F. // Phys. Fluids. 2006. V. 18. N 027104. doi 10.1063/1.2175152

[30] Gromov E.M. and Malomed B.A. Emulating the Raman Physics in the Spatial Domain with the Help of the Zakharov's Systems. Generalized Models and Non-classical Approaches in Complex Materials 2. Structured Materials. V. 90. Springer, Cham, 2018.
[31] Forest M.G. and Lee J.E. in Oscillation Theory, Computation, and Methods of Compensated Compactness. ed. by C. Dafermos et al. IMA Volumes on Mathematics and its Applications 2. Springer, New York. 1987.]

[32] Павлов М.В. // Теоретическая и математическая физика. 1987. T. 71 . C. $351-356$. 